Ken Nakano, Valentin L. Popov

\title{
Dynamic stiction without static friction: The role of friction vector rotation
}

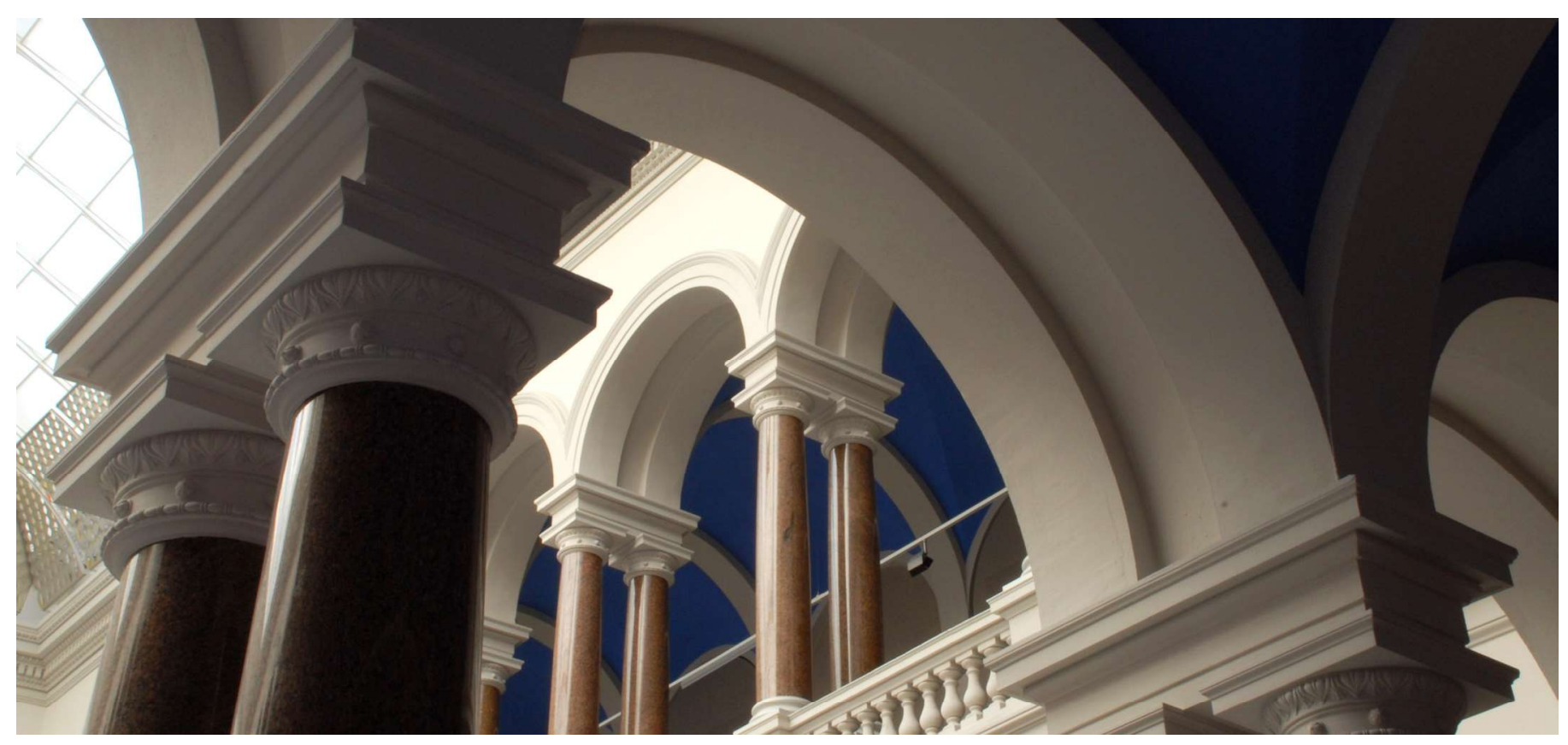

Nakano, K., \& Popov, V. L. (2020). Dynamic stiction without static friction: The role of friction vector rotation. Physical Review E, 102(6). https://doi.org/10.1103/physreve.102.063001 


\title{
Dynamic Stiction without Static Friction: The Role of Friction Vector Rotation
}

\author{
Ken Nakano ${ }^{1} \&$ Valentin L. Popov ${ }^{2}$ \\ ${ }^{1}$ Yokohama National University, Yokohama 240-8501, Japan \\ ${ }^{2}$ Technische Universität Berlin, Berlin 10623, Germany
}

\begin{abstract}
In the textbook formulation of dry friction laws, static and dynamic friction (stick and slip) are qualitatively different and sharply separated phenomena. However, accurate measurements of stick-slip motion generally show that static friction is not truly static but characterized by a slow creep that, upon increasing tangential load, smoothly accelerates into bulk sliding. Microscopic, contact-mechanical, and phenomenological models have been previously developed to account for this behavior. In the present work, we show that it may instead be a systemic property of the measurement apparatus. Using a mechanical model that exhibits the characteristics of typical setups of measuring friction forces - which usually have very high transverse stiffness - and assuming a small but nonzero misalignment angle in the contact plane, we observe some fairly counterintuitive behavior: Under increasing longitudinal loading, the system almost immediately starts sliding perpendicularly to the pulling direction. Then the friction force vector begins to rotate in the plane, gradually approaching the pulling direction. When the angle between the two becomes small, bulk sliding sets in quickly. Although the system is sliding the entire time, macroscopic stick-slip behavior is reproduced very well, as is the accelerated creep during the "stick" phase. The misalignment angle is identified as a key parameter governing the stick-to-slip transition. Numerical results and theoretical considerations also reveal the presence of high-frequency transverse oscillations during the "static" phase, which are also transmitted into the longitudinal direction by nonlinear processes. Stability analysis is carried out and suggests dynamic probing methods for the approaching moment of bulk slip and the possibility of suppressing stick-slip instabilities by changing the misalignment angle and other system parameters.
\end{abstract}

\section{INTRODUCTION}

Dry friction plays an essential role in a great variety of physical processes and numerous engineering, geological, biological, medical, and metrological applications [1-3]. One of the central properties of dry friction is a sharp transition from the static state (stick) to dynamic state (slip): If an increasing lateral force is applied to an object, it will remain at rest while the force is below some threshold value called static friction. When the threshold is met, the object abruptly begins to slide. This property has even prompted the development of a special branch of mechanics - dynamics of systems with non-smooth interactions $[4,5]$. However, more accurate measurements consistently show that there is no discrete transition. As the lateral force increases, the object experiences, from the macroscopic point of view, a continuous creeping motion, which accelerates smoothly as the threshold is approached, and then seamlessly transitions into bulk sliding (while the true microscopic dynamics can still remain discontinuous) [6,7]. This accelerated creep effect not only qualitatively changes the physical picture of static friction, but also plays an important role in earthquake dynamics [8] and in the tribological aspects of many modern technologies such as robotics [9], micromechanics [10] and precision positioning systems [11].

How should accelerated creep be explained? One possibility is that the effect is rooted in macroscopic contact mechanics: If a spherical contact - the perennial favorite in friction experiments - is loaded tangentially, sliding does not affect the entire contact area at once, but rather propagates from the edge inwards as the tangential load increases.
Because normal stress is zero at the edge of a non-adhesive contact, there is no threshold value for the onset of partial slip, and the acceleration of creep as more and more of the contact begins to slide is consistent with experimental data. A prominent representative of this approach is the theory of partial sliding by Cattaneo [12] and Mindlin [13]. It was mostly investigated in the context of fretting wear [14] and frictional damping [15], but it was only recently considered as an explanation for frictional creep [16].

Another possibility is to look for answers at the microscale. It is not implausible to imagine, for example, that some of the weaker "asperity bridges" in the contact start to detach at a much lower force than is necessary for bulk sliding. As the tangential load increases, the rate of such detachments might accelerate and ultimately grow into an avalanche that precipitates macroscopic slip [17]. The process may also be thermally activated. This approach is embodied in the rateand state-dependent laws of friction developed in the 1970s in the context of geophysics $[18,19]$. A similar, but purely phenomenological approach has been developed in the context of pre-sliding $[11,20]$. Further approaches combining both of the above views exist as well, e.g., rapid propagation of slip or detachment fronts in the contact plane [21]. While details vary, the currently predominant view in the tribological community seems to be that creep or pre-sliding is an intrinsic property of friction and as such is caused by some micro-scale mechanism.

Here we present evidence that in typical experimental devices used for studying friction, an approach may be possible, which differs from both above approaches. Instead, in the cases described in the present paper, both apparent 
stick and accelerated creep appear to be a property of the measurement apparatus itself. The strong stiffness anisotropy typical of tribological apparatus using leaf springs, combined with subtle misalignment (possibly less than one degree) between the direction of motion and the supporting spring, causes the friction force vector to rotate in the contact plane, which influences the time evolution of observed displacement. This paves the way for a purely macroscopic mechanical theory of static friction, slow creep, and the stickto-slip transition.

The importance of the rotation of the direction of friction force was previously highlighted in [22] in the context of active control of friction by transverse oscillations. The apparent stick due to friction vector rotation is described in greater detail in the present paper.

\section{MODEL}

Consider a simple mass-spring system as shown in Fig. 1. Sliding systems are usually depicted in a "side view" (Fig. 1a) where a rigid object of mass $m$ is coupled to a spring and is in contact with a horizontal flat floor ( $X Y$ plane) under normal load $W$. The right end of the spring is driven along the $X$-axis with drive velocity $V$. However, for describing the rotation of the friction force vector $\boldsymbol{F}$, we need a "top view" of the system (Fig. 1b). In this projection, the spring is characterized by the stiffness tensor. Let $x$ and $y$ be the principal axes of the stiffness tensor (as shown with green dashed lines in Fig. 1b) and $k_{x}$ and $k_{y}$ the corresponding principal values. We assume that the principal axes are inclined relative to the direction of the drive velocity by a small angle $\varphi$. Such a finite misalignment angle is not an unrealistic assumption because exact alignment between the two axes in the tribological apparatus (and probably, even in any other real sliding system) is virtually impossible. The projections of the spring forces onto the principal axes are $k_{x} u_{x}$ and $k_{y} u_{y}$, where $u_{x}$ and $u_{y}$ are the spring elongations in the $x$ and $y$ directions, respectively. The velocity of the immediate contact point relative to the stationary floor will be denoted as slip velocity $\boldsymbol{v}_{\text {slip. }}$. The direction of the friction force $\boldsymbol{F}$ is assumed to be opposite to the direction of $\boldsymbol{v}_{\text {slip }}$ and its magnitude a continuous function of the magnitude of $\boldsymbol{v}_{\text {slip: }}$ : $F=F\left(v_{\text {slip }}\right)$. Two different laws of friction are used: (i) "velocity-weakening friction" in Section III, determined by $F=\left[\mu_{\infty}+\left(\mu_{0}-\mu_{\infty}\right) \exp \left(-v_{\text {slip }} / v_{\mathrm{f}}\right)\right] W\left(\right.$ where $\mu_{0}$ and $\mu_{\infty}$ are the friction coefficients for $v_{\text {slip }} \sim 0$ and $\infty$, respectively, and $v_{\mathrm{f}}$ is a velocity constant) and (ii) "constant friction" in Sections IV and $\mathrm{V}$, determined by $F=F_{0}=\mu_{0} W$. Note that velocity weakening is not the only mechanism of instabilities in sliding systems. In [23], it was shown that rate- and statedependent friction can also lead to stick-slip movement. Also note that we have left the law of static friction unspecified. Although the resulting system dynamics shows what appear to be stick phases, the frictional contact is in a permanent state of slip.

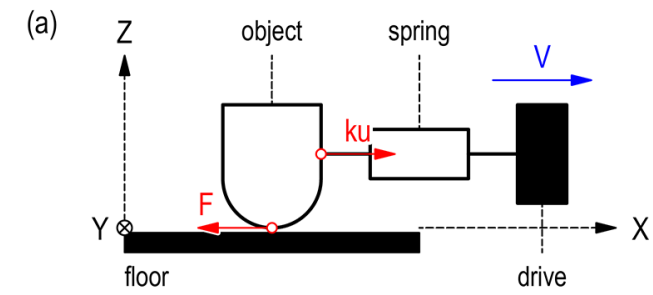

(b)

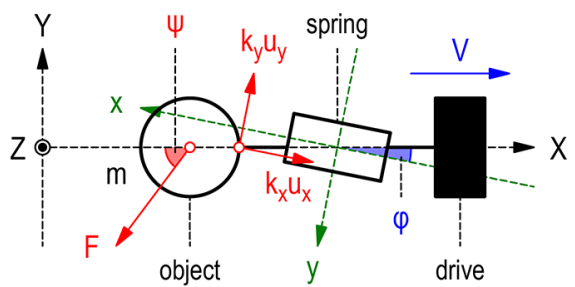

(c)

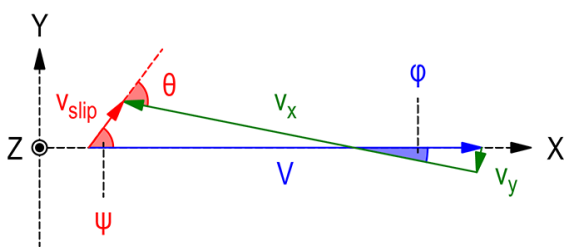

FIG. 1. Analytical model. (a) Side view: An object in contact with a horizontal floor is pulled to the right via a spring. (b) Top view: A non-zero in-plane misalignment $\varphi$ exists between the pulling direction $X$ and the principal axis $x$ of the stiffness tensor. (c) Geometrical relationship of velocities (drive velocity, $V$, spring elongation rates, $v_{x}\left(=\dot{u}_{x}\right)$ and $v_{y}\left(=\dot{u}_{y}\right)$, and slip velocity, $\left.v_{\text {slip }}\right)$ and angles $(\varphi, \psi$, and $\theta)$. The angle $\psi$ of friction force $\boldsymbol{F}$ in (b) is determined from the direction of $\boldsymbol{v}_{\text {slip }}$ in (c). Note that $\theta=\psi+\varphi$.

The equations of motion of the mass $m$ in coordinates $x$ and $y$ read:

$m \ddot{u}_{x}+k_{x} u_{x}=F \cos \theta, \quad m \ddot{u}_{y}+k_{y} u_{y}=F \sin \theta$,

where $\theta=\psi+\varphi$ is the angle between the friction force and the $x$-axis. From the geometry represented in Fig. 1c, one can derive

$\cos \theta=\frac{V \cos \varphi-\dot{u}_{x}}{v_{\text {slip }}}, \sin \theta=\frac{V \sin \varphi-\dot{u}_{y}}{v_{\text {slip }}}$

with

$v_{\text {slip }}=\sqrt{\left(V \cos \varphi-\dot{u}_{x}\right)^{2}+\left(V \sin \varphi-\dot{u}_{y}\right)^{2}}$.

With account of Eqs. (2) and (3), Eq. (1) can be rewritten as

$$
\begin{aligned}
& m \ddot{u}_{x}+k_{x} u_{x}=F\left(v_{\text {slip }}\right) \frac{V \cos \varphi-\dot{u}_{x}}{\sqrt{\left(V \cos \varphi-\dot{u}_{x}\right)^{2}+\left(V \sin \varphi-\dot{u}_{y}\right)^{2}}}, \\
& m \ddot{u}_{y}+k_{y} u_{y}=F\left(v_{\text {slip }}\right) \frac{V \sin \varphi-\dot{u}_{y}}{\sqrt{\left(V \cos \varphi-\dot{u}_{x}\right)^{2}+\left(V \sin \varphi-\dot{u}_{y}\right)^{2}}} .
\end{aligned}
$$

This system of two non-linear second-order differential equations completely determines the dynamics of the system. They were solved numerically using the Runge-Kutta 
method. The time evolution of object position in the laboratory coordinates can be obtained by

$X=V t-u_{x} \cos \varphi-u_{y} \sin \varphi, \quad Y=u_{x} \sin \varphi-u_{y} \cos \varphi$

and

$\dot{X}=V-\dot{u}_{x} \cos \varphi-\dot{u}_{y} \sin \varphi, \quad \dot{Y}=v_{x} \sin \varphi-v_{y} \cos \varphi$.

\section{SYSTEM DYNAMICS FOR VELOCITY- WEAKENING FRICTION}

Fig. 2 presents numerical solutions to the equations of motion for velocity-weakening friction. A small in-plane misalignment of $\varphi=1^{\circ}$ and a strong stiffness anisotropy of $k_{y} / k_{x}=10^{4}$ were assumed corresponding to typical leaf springs used in laboratory friction tests. Other parameters are listed in the caption.
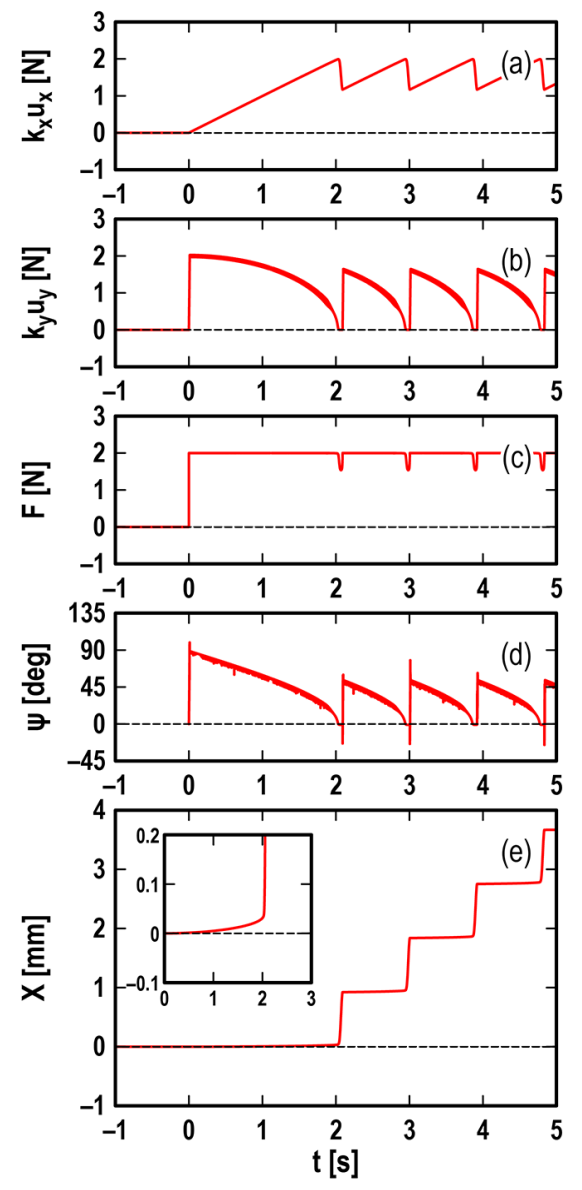

FIG. 2. Dynamic stiction, slow creep, and stick-slip under velocity-weakening friction. (a) Spring force, $k_{x} u_{x}$. (b) Spring force, $k_{y} u_{y}$. (c) Magnitude of friction force, $F$. (d) Direction of friction force, $\psi$. (e) Object position, $X$. System parameters: $\varphi=1^{\circ}, m=0.25$ $\mathrm{kg}, k_{x}=1 \mathrm{kN} / \mathrm{m}, k_{y}=10 \mathrm{MN} / \mathrm{m}, \mu_{0}=0.20, \mu_{\infty}=0.15, v_{\mathrm{f}}=10 \mathrm{~mm} / \mathrm{s}$, $W=10 \mathrm{~N}$, and $V=0$ for $t<0$ and $1 \mathrm{~mm} / \mathrm{s}$ for $t \geq 0$. Initial conditions: $X(0)=0, Y(0)=0, \quad \dot{X}(0)=\varepsilon V$, and $\dot{Y}(0)=0$, where $\varepsilon=10^{-6}$.

The drive started to move at $t=0$. The time dependencies of the $x$ - and $y$-components of the spring force are shown in Figs. $2 \mathrm{a}$ and $2 \mathrm{~b}$, respectively. The longitudinal component of the spring force shows the classical stick-slip behavior consisting of a linear increase in time followed by a sharp drop (Fig. 2a). The transverse component of the force, on the contrary, reveals an unexpected behavior: It jumps to the maximum value (equal to the magnitude of the friction force at low sliding velocity) and subsequently decreases to vanish at the start of the slip phase (Fig. 2b). The magnitude of the force remains practically constant during the whole stick phase (Fig. 2c) dropping only in the phases of rapid slip. Equilibrium in the pulling direction is maintained by the inplane rotation of the friction force vector described by the angle $\psi$ (Fig. 2d). The time dependency of the longitudinal coordinate $X$ (Fig. 2e) shows a pronounced stick-slip character. Although the stair-like object position $(X)$ and the sawtooth-shaped spring force $\left(k_{x} u_{x}\right)$ indicate typical stick-slip, in reality, the object never comes to a full stop. During the stick phases, the object is slowly slipping and gradually accelerating in the $X$ direction (see the inset of Fig. 2e), which is reminiscent of the so-called "slow creep" known from studies of the rate- and state-dependent friction laws [6]. To underline the dynamic nature of the apparent stick phase we call it "dynamic stiction".
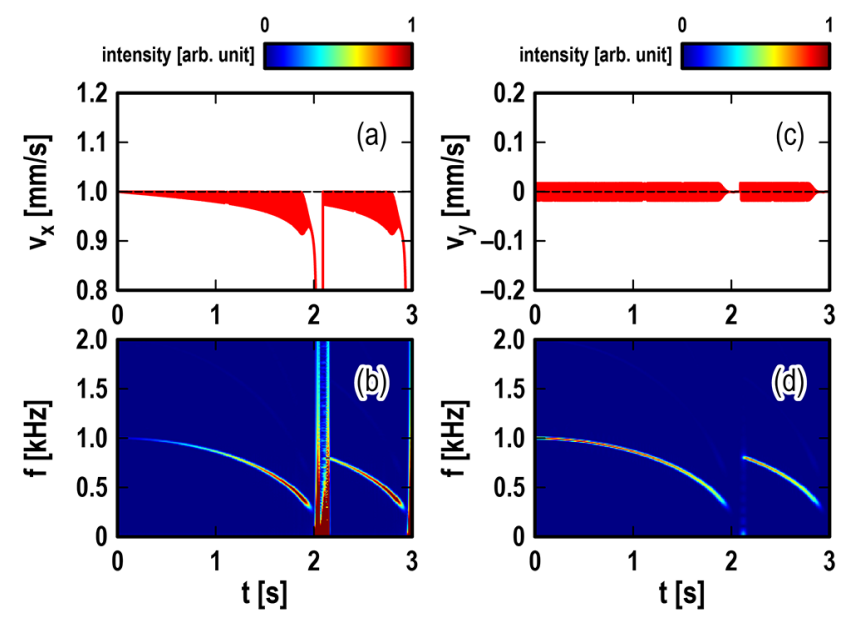

FIG. 3. High-frequency oscillations. (a) Spring elongation rate, $v_{x}\left(=\dot{u}_{x}\right)$, and (b) its spectrogram. (c) Spring elongation rate, $v_{y}(=$ $\dot{u}_{y}$ ), and (d) its spectrogram. System parameters and initial conditions are the same as in Fig. 2. The spectrograms have been obtained using short-time Fourier transform with Hamming window of the width $0.1 \mathrm{~s}$.

The dynamic nature of stiction has important physical implications. One of them is the presence of high-frequency oscillations during the "stick" phase. These oscillations, shown in Fig. 3, are an inherent property of the described system, and will be discussed in more detail below. Spectral analysis shows that the oscillation frequency initially coincides with the natural frequency of transverse 
oscillations, $\omega_{y}=\left(k_{y} / m\right)^{1 / 2}$ (for the system parameters used, $1.0 \mathrm{kHz}$ ) and decreases when approaching the phase of rapid slip (Figs. 3b and 3d). Although both transverse and longitudinal oscillations are present, the fact that they have a common frequency coinciding with the transverse natural frequency indicates that the oscillations originate in the transverse degree of freedom, $y$, and are transmitted to longitudinal movement over a non-linear coupling.

The high-frequency dynamics of sliding systems is of significant interest for many technical applications [24,25]. Their physical origin and influencing factors have been studied for many decades, but their nature often remains unclear [25]. The concept of friction vector rotation offers a new perspective on this phenomenon.

\section{SYSTEM DYNAMICS FOR CONSTANT FRICTION}

Fig. 4 presents results for constant sliding friction. The first transition from "stick" to "slip" is in this case almost identical to the case of velocity-weakening friction; however, no periodic stick-slip motion takes place.

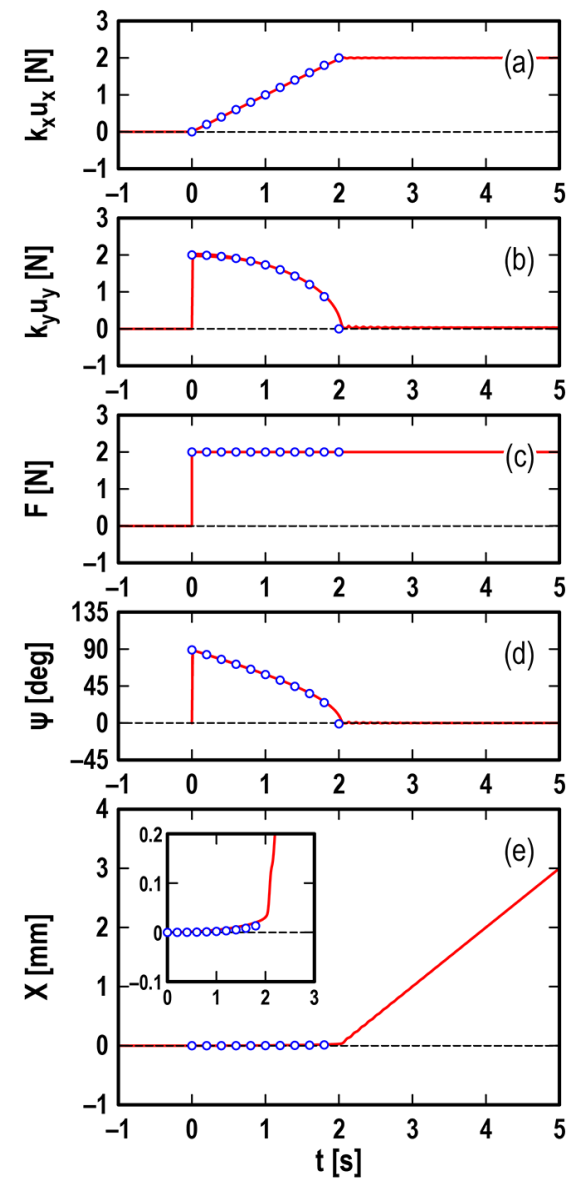

FIG. 4. Dynamic stiction and single stick-to-slip transition under constant friction. (a) Spring force, $k_{x} u_{x}$. (b) Spring force, $k_{y} u_{y}$. (c) Magnitude of friction force, $F$. (d) Direction of friction force, $\psi$. (e)
Object position, $X$. System parameters: $\varphi=1^{\circ}, m=0.25 \mathrm{~kg}, k_{x}=1$ $\mathrm{kN} / \mathrm{m}, k_{y}=10 \mathrm{MN} / \mathrm{m}, \mu_{0}=0.20, W=10 \mathrm{~N}$, and $V=0$ for $t<0$ and $1 \mathrm{~mm} / \mathrm{s}$ for $t \geq 0$. Initial conditions: $X(0)=0, Y(0)=0, \dot{X}(0)=\varepsilon V$, and $\dot{Y}(0)=0$, where $\varepsilon=10^{-6}$. The blue circles are the approximate solutions provided by equations in the text, where those in (e) have been obtained by numerical integration of Eq. (13).

The detailed character of the friction law seemingly has no influence on the phenomenology of the stick-to-slip transition. In retrospect, this is fairly obvious, as the whole creep phase occurs at very small velocity; the velocity dependence is thus mostly of interest during the phase of rapid slip. However, it also determines the character of highfrequency oscillations during the apparent stick phase. With constant friction, the oscillations attenuate more rapidly compared with the case of velocity-weakening friction law.

The character of the friction law exerts its greatest influence on the transition from slip back to stick. While the velocity-weakening law leads to periodic stick-slip (Fig. 2), the sliding phase continues indefinitely in the case of constant friction (Fig. 4). Accordingly, all features related to the back transition from slip to stick are absent in the case of constant friction. Thus, no drop of the friction force appears during the slip phase (compare Fig. 2c and Fig. 4c) because the rapid drop of the magnitude of friction in Fig. 2c is obviously caused by the velocity weakening of friction force.

In the following, we focus only on the stick-to-slip transition, which is very similar for both types of friction law. As all described features are also observed with constant friction, we focus on this simple case to avoid unnecessary complications. We discuss in more detail the main features of the observed stick-to-slip transition: (a) dynamic stiction, (b) slow creep, (c) high-frequency dynamics, and (d) lowfrequency dynamics.

\section{A. Dynamic stiction}

From the results presented so far, we would like to draw the following qualitative picture of dynamic stiction. Dynamic stiction starts with a jump-like rotation of the vector of friction force to the direction almost perpendicular to the sliding direction $\left(\psi=90^{\circ}\right.$, see Fig. $\left.4 \mathrm{~b}\right)$ followed by a gradual decrease to $\psi=0$. This somewhat counterintuitive behavior arises due to the strong anisotropy of the spring, which amplifies the small projection of the spring elongation onto the $y$-direction to such a degree that it completely dominates the friction force vector in the initial stage of dynamic stiction. During subsequent pulling, the $x$-component of the spring force continues to increase, but since the system is already sliding with a very small velocity, the absolute value $F=\left(F_{x}{ }^{2}\right.$ $\left.+F_{y}^{2}\right)^{1 / 2}$ of the force vector remains constant and equal to $F_{0}$ $=\mu_{0} W$ (Fig. 4c). Thus, the perpendicular component of the friction force, $F_{y}=\left(F_{0}^{2}-F_{x}^{2}\right)^{1 / 2}$, is decreasing, gradually approaching zero (Fig. 4b). The change in the pulling force can be supported by the rotation of the friction force vector 
only while $F_{x} \leq F_{0}$. As soon as the pulling force exceeds this critical value, the quasi-static equilibrium breaks down, and the phase of rapid slip starts.

Thus, even though the system is sliding the entire time, the macroscopic dynamics is strongly reminiscent of classical stick-slip.

\section{B. Slow creep}

Let us consider in more detail the "stick" phase (which in reality is a phase of slow creep). The movement during this stage is quasistatic, which means that the inertial terms can be neglected. However, this is valid only for the movement in the $x$-direction. The high transverse stiffness $k_{y}$ guarantees very small deflections $u_{y}$. The velocity $\dot{u}_{y}$ is not necessarily small due to the high natural frequency in the $y$-direction, but it has zero average and can be set to zero while considering the creep process. Thus, in the creep phase (and only for the creeping part of the motion), we can neglect in Eqs. (4) and (5) the terms with $\ddot{u}_{x}$ and $\dot{u}_{y}$. After some transformations, this leads to

$\dot{u}_{x}=V \cos \varphi-\frac{k_{x} u_{x} / F_{0}}{\sqrt{1-\left(k_{x} u_{x} / F_{0}\right)^{2}}} V \sin \varphi$.
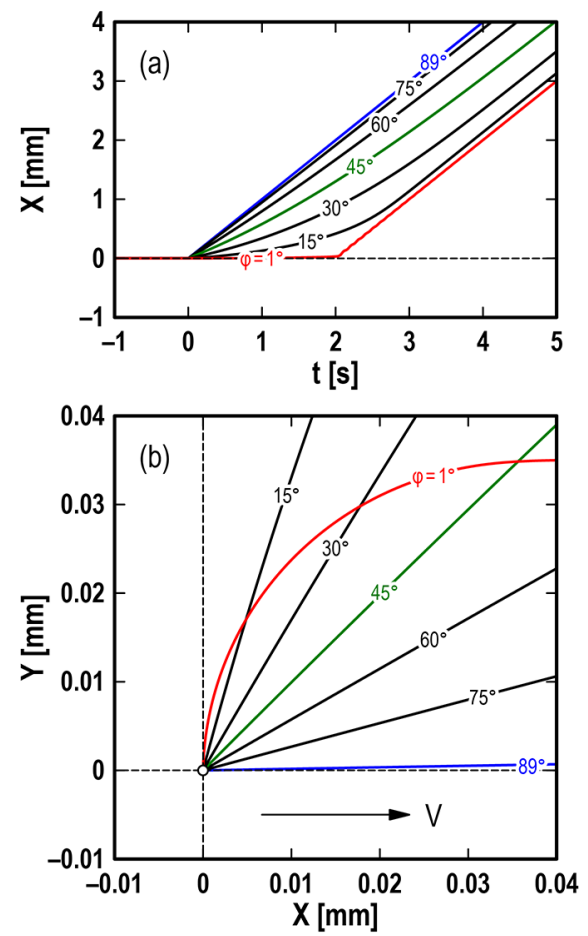

FIG. 5. Disappearance of dynamic stiction with large in-plane misalignment. (a) Temporal changes in object position $X$ for various misalignment angles $\varphi$ from $1^{\circ}$ to $89^{\circ}$. (b) Trajectories of object position in the $X Y$ plane. System parameters (except $\varphi$ ) and initial conditions are the same as in Fig. 4.

This is an ordinary differential equation of the first order, which completely determines the dynamics of the degree of freedom $u_{x}(t)$. The coordinate $X$ in the driving direction can finally be found using Eq. (6). The resulting solutions almost exactly coincide with solutions of the complete dynamic equations (4) and (5) shown in Fig. 5. One can see that at small misalignment, the system shows almost perfect stick, which rapidly becomes blurred when the misalignment angle increases.

In the limiting case of very small misalignment angles, Eq. (8) takes the form $\dot{u}_{x} \approx V$ with the solution $u_{x}=V t$ (for $V t$ $<l_{x}=F_{0} / k_{x}$ ). Thus, the longitudinal and transverse spring forces will be respectively equal to:

$k_{x} u_{x}=k_{x} V t$ for $k_{x} V t<F_{0}$

and

$k_{y} u_{y}=\sqrt{F_{0}^{2}-\left(k_{x} V t\right)^{2}}$ for $k_{x} V t<F_{0}$.

The angle $\theta$ between the force and $x$-direction is determined by the equation $\cos \theta=k_{x} u_{x} / F_{0}=V t / l_{x}$ or

$\theta=\arccos \left(\frac{V t}{l_{x}}\right)$.

For the angle $\psi$, we have

$\psi=\theta-\varphi$.

Dependencies described by Eqs. (9)-(11) with Eq. (12) are shown in Fig. 4 with blue circles together with the numerical result for $\varphi=1^{\circ}$.

For the creep velocity, Eq. (8) with Eq. (7) yields

$\dot{X} \approx V-\dot{u}_{x} \approx V \varphi\left[\varphi+\frac{\left(V t / l_{x}\right)^{2}}{\sqrt{1-\left(V t / l_{x}\right)^{2}}}\right]$.

This creep dependence is shown in the inset of Fig. 4e together with results of full dynamic simulation. It contains two contributions: One of the first order in pulling velocity and of second order in misalignment angle; and a second one that is linear in misalignment angle and second order in the pulling velocity. The second term has a singularity of the form $\left[1-\left(V t / l_{x}\right)^{2}\right]^{-1 / 2}$ when approaching the moment of "slip". Comparison with published measurements of creep [7] seems to confirm the existence of these two contributions. Our simulations suggest that the angle of misalignment is a crucial parameter determining the creep rate. From Eq. (13), one can estimate that the creep velocity achieves the order of the pulling velocity when $\left(V t-l_{x}\right) / l_{x}=(1 / 2) \varphi^{2}$. This quantity characterizes the relative size of the "blurred region" compared to the total time of "stick". It is determined solely by the misalignment angle.

Fig. $5 \mathrm{~b}$ shows the trajectory of the object during the stick phase. It is seen that at a small misalignment angle of $1^{\circ}$, the object starts moving in the direction perpendicular to the pulling direction and achieves a saturation level, whose theoretical value is $\left(F_{0} / k_{x}\right) \sin \varphi \cos \varphi(0.035 \mathrm{~mm}$ for the system parameter used). During this process, the direction of sliding, $\psi$, rotates from $\pi / 2-\varphi$ to 0 . 


\section{High-frequency dynamics}

At the beginning of the creep phase, $V \cos \varphi-\dot{u}_{x} \approx 0$. Neglecting this term in Eq. (5) transforms this equation to

$m \ddot{u}_{y}+k_{y} u_{y}+F_{0} \operatorname{sign}\left(\dot{u}_{y}-V \sin \varphi\right)=0$.

The average value of displacement is easily found by setting $\dot{u}_{y}=0$ and $\ddot{u}_{y}=0: k_{y}\left\langle u_{y}\right\rangle=F_{0}$, while the amplitude of oscillations is determined by the non-linear term $F_{0} \operatorname{sign}\left(\dot{u}_{y}-V \sin \varphi\right)$. Multiplying Eq. (14) with $\dot{u}_{y}$, we can rewrite it as $\mathrm{d} E / \mathrm{d} t+F_{0} \dot{u}_{y} \operatorname{sign}\left(\dot{u}_{y}-V \sin \varphi\right)=0$, where $E$ is the energy of the system. Averaging over one oscillation period yields

$\left\langle\frac{\mathrm{d} E}{\mathrm{~d} t}\right\rangle=-F_{0}\left\langle\dot{u}_{y} \operatorname{sign}\left(\dot{u}_{y}-V \sin \varphi\right)\right\rangle$.

The result is negative if the amplitude of oscillation, $\dot{\bar{u}}_{y}$, is larger than $V \sin \varphi$ and becomes zero when it is equal to $V \sin \varphi$. After that, the system shows undamped oscillations with the constant amplitude

$\dot{\bar{u}}_{y}=V \sin \varphi$

and frequency $\omega_{y}=\left(k_{y} / m\right)^{1 / 2}$. The oscillation amplitude is equal to the $y$-component of the drive velocity. Note that in the case of friction with a constant magnitude, this is the upper bound of the oscillation amplitude. In the further course of creep, Eq. (14) is not strictly valid, and some damping appears so that finally the oscillations die out (see a more detailed analysis of damping in Section V). In the case of velocity-weakening friction, oscillations of small amplitude are amplified until they reach the value of Eq. (16), which in this case has the meaning of amplitude of stable limiting cycle. The above-stated difference between constant and velocity-weakening friction can be seen by comparison of Fig. $2 b$ and Fig. $4 b$.

\section{Low-frequency dynamics}

Let us now consider oscillations in the longitudinal direction (along the $x$-axis) in the slip phase. In the full dynamic equation (4), we can now neglect $\dot{u}_{y}$ as a small variable and consider the Taylor series expansion up to the first order in $\dot{u}_{x}$ :

$\ddot{u}_{x}+2 \delta \cdot \dot{u}_{x}+\omega_{x}^{2} u_{x}=0$

with $\omega_{x}=\left(k_{x} / m\right)^{1 / 2}$ and $\delta=F_{0} \sin ^{2} \varphi / 2 m V$. This equation describes oscillation with frequency $\left(\omega_{x}^{2}-\delta^{2}\right)^{1 / 2}$ and exponentially decreasing amplitude, $\exp (-\delta t)$. The condition for an overdamped system reads $\delta>\omega_{x}$ or $F_{0} \sin ^{2} \varphi / 2 V(m k x)^{1 / 2}$ $>1$. For the system parameters described in the caption of Fig. 4, this means $\varphi>7.2^{\circ}$.

In Fig. 6a, one can see slowly attenuating oscillations at $\varphi$ $=1^{\circ}$ (red curve) and overdamped motion without oscillations at $\varphi=10^{\circ}$. In other words, large misalignment angles suppress frictionally induced oscillations in the pulling direction $[26,27]$. At the same time, they can facilitate oscillations in the transverse direction as can be seen in Fig. 6b. A detailed stability analysis is carried out in Section V.

Note that the rotation of the friction force vector is a common feature characteristic for both small and large misalignments (Fig. 6c).

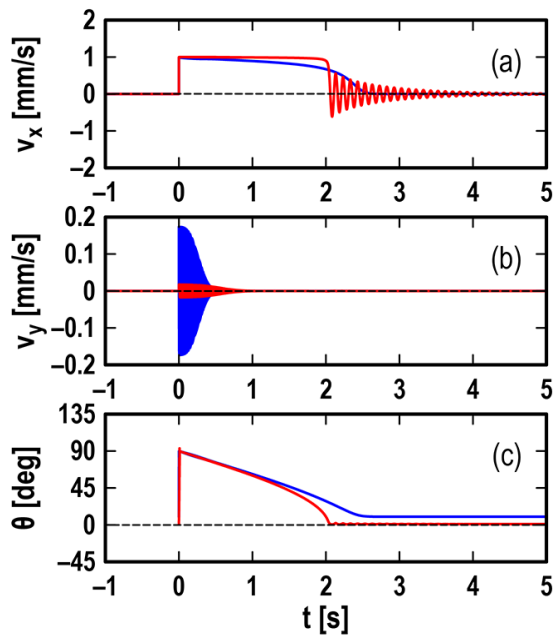

FIG. 6. Suppression of low-frequency oscillations and enhancement of high-frequency oscillations by increasing in-plane misalignment. (a) Spring elongation rates, $v_{x}\left(=\dot{u}_{x}\right)$. (b) Spring elongation rate, $v_{y}\left(=\dot{u}_{y}\right)$. (c) Direction of friction force, $\theta(=\psi+\varphi)$. Red and blue lines are numerical results for $\varphi=1^{\circ}$ and $10^{\circ}$, respectively. System parameters (except $\varphi$ ) and initial conditions are the same as in Figs. 4 and 5.

\section{STABILITY ANALYSIS}

To obtain a general view of the dynamic properties of the considered system, let us undertake a linear stability analysis. We consider the stability of the state $\dot{u}_{y}=0$ (where the average velocity $\dot{u}_{x}$ may vary significantly, but very slowly). Equations for small deviations $\tilde{u}_{x}$ and $\tilde{u}_{y}$ can be obtained by expanding the equations of motion (4) and (5) up to the terms of the first order in perturbations of displacement, velocity, and acceleration and setting $\dot{u}_{v}=0$ :

$\ddot{\tilde{u}}_{x}+\omega_{x}^{2} \tilde{u}_{x}+\alpha \sin ^{3} \theta \cdot \dot{\tilde{u}}_{x}-\alpha \cos \theta \sin ^{2} \theta \cdot \dot{\tilde{u}}_{y}=0$

$\ddot{\tilde{u}}_{y}+\omega_{y}^{2} \tilde{u}_{y}-\alpha \cos \theta \sin ^{2} \theta \cdot \dot{\tilde{u}}_{x}+\alpha \cos ^{2} \theta \sin \theta \cdot \dot{\tilde{u}}_{y}=0$

with

$\alpha=\frac{F_{0}}{m V \sin \varphi}$.

Searching for a solution of the form $\tilde{u}_{x}, \tilde{u}_{y} \propto \exp (\lambda t)$, we find for $\lambda$ the following characteristic equation:

$$
\begin{aligned}
& \lambda^{4}+\lambda^{3} \alpha \sin \theta+\lambda^{2}\left(\omega_{x}^{2}+\omega_{y}^{2}\right) \\
& \quad+\lambda \alpha\left[\left(\omega_{x}^{2}-\omega_{y}^{2}\right) \sin \theta \cos ^{2} \theta+\omega_{y}^{2} \sin \theta\right]=0
\end{aligned}
$$

Assuming $\lambda_{1} \approx-i \omega_{x}-\Delta_{x}$ and $\lambda_{2} \approx-i \omega_{y}-\Delta_{y}$ and substituting both into Eq. (21), we find for small attenuation 
$\Delta_{x}=\frac{\alpha}{2} \sin ^{3} \theta, \quad \Delta_{y}=\frac{\alpha}{2} \sin \theta \cos ^{2} \theta$.

The oscillations in the $x$ - and $y$-directions become overdamped when the damping factors $\zeta_{x}$ and $\zeta_{y}$ exceed unity:

$$
\zeta_{x}=\frac{\Delta_{x}}{\omega_{x}}=\Lambda_{x} \sin ^{3} \theta>1, \quad \zeta_{y}=\frac{\Delta_{y}}{\omega_{y}}=\Lambda_{y} \sin \theta \cos ^{2} \theta>1
$$

where

$$
\Lambda_{x}=\frac{\mu_{0} W}{2 V \sin \varphi \sqrt{m k_{x}}}, \quad \Lambda_{y}=\frac{\mu_{0} W}{2 V \sin \varphi \sqrt{m k_{y}}} .
$$

Note that $\Lambda_{x}$ and $\Lambda_{y}$ are dimensionless parameters determined by system parameters.

Consider first the dynamic properties in the longitudinal direction. They are determined by the factor $\zeta_{x}=\Lambda_{x} \sin ^{3} \theta$. This dependency is demonstrated by the damping diagram, Fig. 7a. During the "stick" phase, the angle $\theta$ of the friction force vector slowly changes from $90^{\circ}$ to $\varphi$. Depending on the combination of $\Lambda_{x}$ and $\varphi$, the system in the "overdamping" region can either reach the "underdamping" region or not. For the red line in Fig. 7a (i.e., $\Lambda_{x}=3.6 \times 10^{3}$ and $\varphi=1^{\circ}$ ), the factor is initially quite high $\left(\zeta_{x}=\Lambda_{x}=3.6 \times 10^{3}\right.$ : overdamping $)$ and drops toward a low value $\left(\zeta_{x}=\Lambda_{x} \sin ^{3} \varphi=1.9 \times 10^{-2}\right.$ : underdamping), across the green line $\left(\zeta_{x}=1\right.$ : critical damping) in the vicinity of the stick-to-slip transition. This overdamping-to-underdamping transition causes free oscillations of low frequency after stick-to-slip transition (see the red line in Fig. 6a). For the blue line in Fig. 7a (i.e., $\Lambda_{x}=$ $3.6 \times 10^{2}$ and $\varphi=10^{\circ}$ ), on the other hand, the factor that is always larger than unity makes the system stay in the overdamping region, which in terms of results, suppresses the free oscillations of low frequency (see the blue line in Fig. 6a). Thus, the factor $\zeta_{x}$, determined by $\Lambda_{x}$ and $\theta$, could be used for dynamic probing of the approaching moment of bulk slip.

Similarly, the dynamic properties in the transverse direction are determined by the factor $\zeta_{y}=\Lambda_{y} \sin \theta \cos ^{2} \theta$. The corresponding damping diagram is presented in Fig. $7 \mathrm{~b}$. One can see that in this case, increasing the misalignment angle tends to transfer the system into the underdamping region, thus facilitating oscillations of high frequency. An example of such a transition is illustrated in Fig. $6 \mathrm{~b}$.
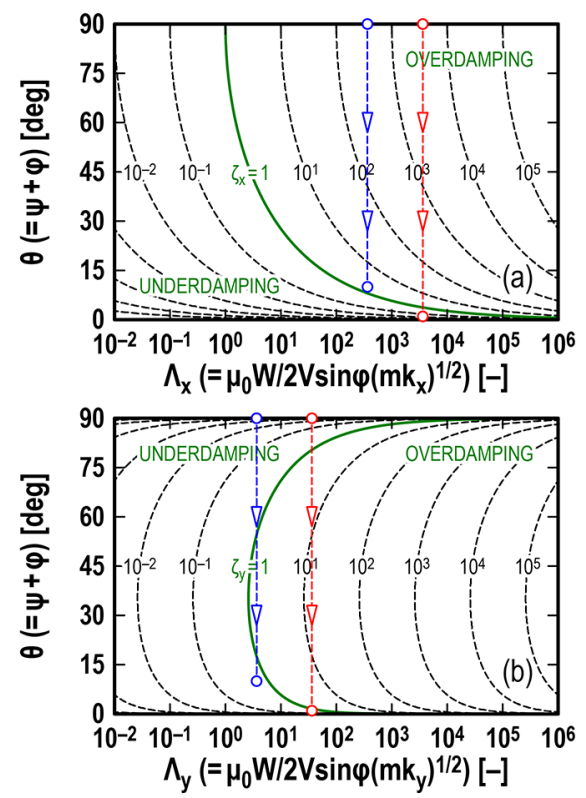

FIG. 7. Damping diagrams. (a) The contour map of damping factor $\zeta_{x}\left(=\Lambda_{x} \sin ^{3} \theta\right)$ in the $x$-direction. (b) The contour map of damping factor $\zeta_{y}\left(=\Lambda_{y} \sin \theta \cos ^{2} \theta\right)$ in the $y$-direction. Green lines denote the critical damping (i.e., $\zeta_{x}=1$ and $\zeta_{y}=1$ in Figs. $7 \mathrm{a}$ and $7 \mathrm{~b}$, respectively). Red and blue lines are trajectories for $\varphi=1^{\circ}$ and $10^{\circ}$, respectively, under the conditions of Fig. 6.

\section{CONCLUSIONS}

We studied the dynamics of a simple mass-spring system, which can be considered representative of typical setups for measuring the force of friction. This or similar experimental configurations were used over centuries starting with the famous tribometer of Coulomb [28] and up to now [29]. For example, the very much cited stick-slip curves reproduced in [30] were obtained on a tribometer using a leaf spring for loading in the sliding direction [31]. All these tribometers have a common structure: A mass, a spring with low stiffness in the sliding direction and high stiffness perpendicular to the sliding direction, a frictional contact, and a pulling mechanism. The behavior of the system is as usual as the system itself: When being slowly pulled, the object first appears to stick and then starts macroscopic sliding.

Analysis of the system is traditionally confined to a single degree of freedom: the movement in the pulling direction [32]. It is easy to intuitively reject the transverse degree of freedom as irrelevant, since the system is "guided", with the very high transverse stiffness preventing any noticeable movement in this direction. Also, any potential misalignment between the pulling direction and the direction of the principal axis of the stiffness tensor is never measured or reported, as it is considered to be small and not likely to be significant.

However, upon closer examination, this logic appears to be faulty. High transverse stiffness in combination with small 
misalignment leads to the immediate start of sliding in the direction almost perpendicular to the pulling direction. The frictional force at the beginning of the pulling process is consequently not zero, as normally assumed, but equal to the friction force in sliding at small velocities. Besides, it is directed not oppositely to the pulling direction but perpendicularly to it! Simulations show that the object is creeping all the time and that the apparent "stick" is due to the rotation of the friction force vector.

This novel interpretation of the apparent stick changes necessarily also the view on slow creep. The rotation of the friction force vector leads to a particular shape of the accelerated creep and reveals the misalignment angle as its main influencing parameter.

Numerical simulations show an additional unexpected phenomenon - high-frequency oscillations perpendicularly to the pulling direction in the "stick" phase, as well as longitudinal oscillations with much lower frequency in the slip phase.

The results offer a new possible view on static friction, slow creep, stick-to-slip transition, and the nature of highfrequency oscillations in sliding systems. Clearly, this is not the only possible mechanism of stick-to-slip transition, as its prerequisite is a high stiffness anisotropy. The present mechanism also does not include (at least so far) the kinetics of friction, as described by the Dieterich-Ruina law.

While being completely aware that the demonstrated mechanism of stick-to-slip transition does not exhaust all possible mechanisms of the stick-slip phenomena, we would like to draw the attention of researchers and engineers to the fact that the well-known and much-debated properties of the transition from stick to slip, including slow creep, may have a completely different - and much simpler - purely mechanical origin.

\section{ACKNOWLEDGMENTS}

The authors acknowledge fruitful discussions with M. Popov.

[1] F. P. Bowden and D. Tabor, The friction and lubrication of solids (Clarendon Press, Oxford, 1986).

[2] B. N. J. Persson, Sliding Friction. Physical Principles and Applications (Springer, 2000).

[3] V. L. Popov, Contact Mechanics and Friction. Physical Principles and Applications. 2nd Ed. (Springer, 2017).

[4] Ch. Glocker, Set-Valued Force Laws. Dynamics of NonSmooth Systems (Springer-Verlag Berlin Heidelberg, 2001).

[5] R. Leine and H. Nijmeijer, Dynamics and Bifurcations of NonSmooth Mechanical Systems (Springer-Verlag Berlin Heidelberg, 2004).

[6] V. L. Popov, B. Grzemba, J. Starcevic, and M. Popov, Rate and state dependent friction laws and the prediction of earthquakes: What can we learn from laboratory models? Tectonophys. 532, 291 (2012).

[7] B. Grzemba, Predictability of Elementary Models for
Earthquake Dynamics. (epubli GmbH, Berlin, 2014).

[8] C. H. Scholz, Earthquakes and Friction Laws, Nature 391, 37 (1998).

[9] C. C. de Wit, H. Olsson, K. Astrom, and P. Lischinsky, A new model for control of systems with friction, IEEE Trans. Autom. Contr. 40, 419 (1995).

[10] M. Zhou, Z. Fan, Z. Ma, H. Zhao, Y. Guo, K. Hong, Y. Li, H. Liu, and D. Wu, Design and Experimental Research of a Novel Stick-Slip Type Piezoelectric Actuator. Micromachines 8, 150 (2017).

[11] V. Lampaert, F. Al-Bender, and J. Swevers, Experimental Characterization of Dry Friction at Low Velocities on a Developed Tribometer Setup for Macroscopic Measurements, Tribol. Lett. 16, 95 (2004).

[12] C. Cattaneo, Sul Contatto di due Corpore Elastici: Distribuzione degli sforzi. Rendiconti Dell'Acad. Nazionale Dei Lincei 27, 342, 434, 474 (1938).

[13] R. D. Mindlin, Compliance of elastic bodies in contact, J. Appl. Mech. 16, 259 (1949).

[14] R. B. Waterhouse, Fretting wear, Wear, 100, 107 (1984).

[15] M. Popov, V. Popov, and R. Pohrt, Relaxation damping in oscillating contacts. Sci. Rep. 5, 16189 (2015).

[16] B. Grzemba, New experimental investigations on the Dieterich-Ruina friction law, Facta Univ. Ser. Mech. Eng. 13, 11 (2015).

[17] M. H. Müser, M. Urbakh, and M. O. Robbins, Statistical Mechanics of Static and Low-Velocity Kinetic Friction. Advances in Chemical Physics, Ed. by I. Prigogine, S.A. Rice, 126, 187 (2003).

[18] J. H. Dieterich, Time Dependent Friction and the Mechanics of Stick-Slip, Pure Appl. Geophys. 116, 790 (1978).

[19] J. R. Rice and A. L. Ruina, Stability of Steady Frictional Slipping, J. Appl. Mech. 50, 343 (1983).

[20] B. Armstrong-Hélouvry, P. Dupont, and C. C. de Wit, Automatica 30, 1083 (1994).

[21] S. M. Rubinstein, G. Cohen, and J. Fineberg, Detachment fronts and the onset of dynamic friction, Nature 430, 1005 (2004).

[22] J. Benad, K. Nakano, V. L. Popov, and M. Popov, Active control of friction by transverse oscillations, Friction 7, 74 (2018).

[23] A. Ruina, Slip instability and state variable friction laws, J. Geophys. Res. Solid Earth 88, 10359 (1983).

[24] J. T. Oden and J. A. C. Martins, Models and computational methods for dynamic friction phenomena, Comput. Methods Appl. Mech. Eng. 52, 527 (1985).

[25] N. M. Kinkaid, O. M. O'Reilly, and P. Papadopoulos, Automotive disc brake squeal, J. Sound Vib. 267, 105 (2003).

[26] N. Kado, C. Tadokoro, and K. Nakano, Measurement error of kinetic friction coefficient generated by frictional vibration, Trans. Jpn. Soc. Mech. Eng. Ser. C 79, 396 (2013).

[27] N. Kado, C. Tadokoro, and K. Nakano, Kinetic friction coefficient measured in tribotesting: influence of frictional vibration, Tribol. Online 9, 63 (2014).

[28] C. A. Coulomb, Theorie des machines simple. (Bachelier, 1821).

[29] M. Heuberger, C. Drummond, and J. Israelachvili, Coupling of Normal and Transverse Motions during Frictional Sliding. The J. Phys. Chem. B, 5038 (1998). 
[30] M. Urbakh, J. Klafter, D. Gourdon, and J. Israelachvili, The nonlinear nature of friction. Nature 430, 525 (2004).

[31] A. Cochard, L. Bureau, and T. Baumberger, Stabilization of Frictional Sliding by Normal Load Modulation. J. Appl. Mech.
70, 220 (2003).

[32] K. Nakano, Two dimensionless parameters controlling the occurrence of stick-slip motion in a 1-DOF system with Coulomb friction, Tribol. Lett. 24, 91 (2006). 